UDC 331.5

http://doi.org/10.21272/mmi.2019.3-25

JEL Classification: L26, M13, E37, C10

Gabriela Opait,

Ph.D., Associate Professor, "Dunarea de Jos» University of Galati, Romania

Daniela Damian,

Ph.D., "Dunarea de Jos» University of Galati, Romania

Alexandru Capatina,

Ph.D., Professor, «Dunarea de Jos» University of Galati, Romania

\title{
FREELANCER AS AN ENTREPRENEUR: A CHOICE AS CAREER DEVELOPMENT
}

Abstract. Entrepreneurship starts to become a challenging option among freelancers worldwide. However, the low rate of entrepreneurship as a career choice among freelancers is due to peculiar factors. Turning freelance activities in sustainable businesses are considered a smart career pathway by many scholars and freelance practitioners. This article aims at revealing through a case study how a freelancer has been immersed into an entrepreneurial approach, his sources of income being influenced by this entrepreneurial mindset shift. This study computes the values of the adjusted informational energy based on inputs for distribution of incomes reported by a Romanian freelancer turned entrepreneur, emphasizing the time series revenues from freelancing, on the one hand, and entrepreneurship, on the other hand. The research is mainly focused on an econometric model, built on informational energy model, highlighting a peculiar decrease of the entropy, as the effect of the higher weights of the incomes from entrepreneurship. Building on mathematical modelling, this study presents an analysis framework that combines financial data and statistical analysis to examine the influence of revenues registered by a freelancer who accepted an entrepreneurial challenge as a second source of income. This study expands previous research by using Onicescu's informational energy theory to a peculiar case of a freelancer turned entrepreneur. The strength of the adjusted informational energy modelling consists of revealing the added value of the statistical analysis and emphasizing the specific architecture of freelancer incomes' influences on the adjusted informational energy. The findings of this research also outline motivation pillars for the freelancers who are willing to commit to entrepreneurial initiatives.

Keywords: freelancing, entrepreneurship, informational energy, entropy, econometrics

Introduction. Freelancers can be considered a hybrid between employees and entrepreneurs. On the one hand, they are employed, because they are almost always employed by companies to work for a while offering only their intangible professional knowledge. This is different from other entrepreneurs and self-employed who sell tangible products to customers. On the other hand, they can be called entrepreneurs because they work at their own risk and for their own reward without any support or organizational guarantee (Van den Born and Van Witteloostuijn, 2013).

This research seeks to provide insights related to a freelancer adoption of an entrepreneurial career from the weights of the revenues achieved by a freelancer turned entrepreneur through Onicescu's (1966) informational energy theory. This study examines the interest of freelancers for an entrepreneurial career by focusing on a specific case study, which explores the distribution of the revenues of a freelancer turned entrepreneur in seven consecutive years. The statistical analyses that this study performs draw on Florea's (1986) Factors Path Method.

After this introduction, section 2 presents a review of the literature on freelancers' careers oriented toward entrepreneurship. Section 3 describes the methodology. Section 4 highlights the influences of the freelancer turned entrepreneur revenues on the dynamics of the adjusted informational energy. Section 5 presents the conclusions of the study.

Literature Review. An independent career can be seen as similar to an unlimited career. DeFillippi and Arthur (2002) have defined unlimited careers as employment opportunity sequences that go beyond the limits of unique vocational training. A freelancer is a person who would change workplace often or

Cite as: Opait, G., Damian, D., Capatina, A. (2019). Freelancer as an Entrepreneur: a Choice as Career Development. Marketing and Management of Innovations, 3, 327-340. http://doi.org/10.21272/mmi.2019.3-25 
the archetypal job hopper, going from one project to another and from one employer to another without leaving too much time in an organization.

Therefore, the concept of boundaryless career offers a good starting point to build a successful model in freelancer career. The concept of a boundaryless career was developed in the 1970s at the Massachusetts Institute of Technology (Tams and Arthur, 2010). However, the prospect of boundaryless career has indeed been developed in the 1990s, particularly through the efforts of Arthur and Rousseau (1996). In the mid-1990s, Arthur, Claman and DeFillippi (1995) highlighted strong links between Quinn's intelligent enterprise and the concept of a boundaryless career. They argue that the foundation of an intelligent enterprise requires an intelligent career that is based on principles from the concept of unlimited career. The intelligent framework of freelancers' careers comprises three classes of interconnected variables, called "ways of knowing», which argue career evolution (Arulmani et al., 2014). The first way to know is to know why and involves career motivation, personal significance, identity and personality. Knowing «why» means the ability of an individual to understand himself or explore different possibilities, to adapt to changing circumstances. «Knowing how» reflects the relevant skills in the career and knowledge of the workplace. «Knowing how» is closely linked to the established ideas on individual knowledge and skills. The third way to know, «know who» involves the relevant personal and business networks. This way of knowing involves networks, contacts, including business relationships and personal connections.

In the literature on entrepreneurial personality, Amit, Glosten and Muller (1993) suggested that the four personality traits representative of activities associated with self-employment: the need for personal achievement, the internal control site, the risk-taking over average and the ambiguity tolerance.

The smart career framework is based initially on organizational models of competencies. This is why the smart career framework provides an appropriate platform for developing the success model of a freelancer career by evaluating the empirical results reported in successful studies looking for the variables to be added to the career model. Adding such business-related issues to its successful model in a freelancer career will create a better match to the challenges and dilemmas in freelancing. After all, a modern freelancer is also an entrepreneur and his own employee without a frontier (Burke, A., \& Van Stel, 2011). That is why the interest is especially focused on the research of small entrepreneurs, such as self-employed and small businesses, so not so much on large enterprises, where the influence of the founders is limited and other qualities are needed for to be successful. Van den Born and Van Witteloostuijn highlights the basic factors of entrepreneurial success identified in the empirical research of self-employed and small businesses.

In the context of freelancing, entrepreneurial initiatives could generate a broad base of recommendations, help the freelancer identify promising opportunities and increase the freelancer's likelihood of knowing how to realize and promote their own project. Thirdly, studying the impact of personal capital on entrepreneurial success has a long tradition. It is claimed that freelancers turned entrepreneurs want to engage everyone's energies, involve many people both inside and outside the organization, create and sustain networking relationships, and take full advantage of intellectual resources and other resources that people offer while helping them achieve their goals (McGrath et al., 2000). These qualities highlight the need for a certain type of personality or other personal characteristics such as self-understanding and leadership. A freelancer turned entrepreneur remains a dilemma because he would waste knowledge and potential earnings that he could earn as a selfemployed person developing his strongest specified skills and accumulating experience and reputation (Backes-Gellner and Moog, 2013).

Freelancers' work experience is crucial for managing organizational complexities in the aspiration toward entrepreneurship, by which is meant start-ups that exhibit motivation for rapid growth (Autio, 
2011), or in knowledge-intensive industries that involve complex technology and previous experience (Unger et al., 2011; Wyrwich, 2013).

As the employment landscape, career research should reflect the entrepreneurial challenges for freelancers, considering their skills favourable to fast mobility between temporary contracts and full-time contracts (Bögenhold et al., 2014). The results of a study conducted by Falco and Haywood (2016) emphasizes that the rise in freelance activities is the result of improved entrepreneurial opportunities or the reflection of limited opportunities in wage-based employment.

Backes-Gellner and Moog (2013) consider that the freelancers who have a sound professional knowledge and innovation capabilities, would not be interested in a great extent to become entrepreneurs because they could waste knowledge and potential earnings. It is difficult to separate the desire of the freelancers to develop entrepreneurship and become involved in R\&D projects (Borowiecki and Makieła, 2019).

Nowadays, plenty of innovative projects carried out by freelancers can be turned into entrepreneurial initiatives via crowdfunding. According to Petrushenko and Dudkin (2014), crowdfunding platforms are cited among the most effective methods of financing modern innovations related to projects focused on Internet and social networks, where the main part of frelancers are doing their jobs. Social entrepreneurship represents another opportunity that can be captured by the freelancers working in this field, considering the strong political incentives in EU level in terms of creating social enterprises (Greblikaite, Sroka and Grants, 2015).

Successful entrepreneurs are ex-freelancers who are able to purposelly built business models, based on their experiences gained from practical environments and move quickly to embrace problemsolving solutions that they have learned through doing (Daum, 2005). When considering the aspect of freelancers' motivation, creativity is helped by encouraging them to submit various ideas, creating an atmosphere of cooperation in their groups, fostering discussions about entrepreneurial initiatives (Lichtarski and Trenkner, 2018).

The motivation to work as a freelancer could be radically changed if entrepreneurial opportunities arise. Freelancers' self-employed work characteristics (expertise, reputation, innovativeness) are strongly related to entrepreneurial skills (Annink et al., 2016). A recent research study analyses configurations of entrepreneurship predictors (experience, reputation, innovation, and idea funding) that enable freelancers to think about a future entrepreneurial career (Damian and Manea, 2019).

Methodology and research methods. The research involves a case study, which reflects the distribution of incomes achieved by a self-employed person (freelancer) turned entrepreneur in seven consecutive years. The freelancers reported the growth of revenues from entrepreneurship, while the incomes from his independent activities decreased in the period of analysis. His main activities as freelancer and entrepreneur, as well, have been carried out in the field of design and development of ecommerce portals, Search Engine Optimization and Search Engine Marketing.

The econometric model involves the configuration of the incomes from freelancing, respectively of the incomes from entrepreneurship, achieved by a Romanian freelancer turned entrepreneur, in the period 2011-2017 (Table 1); these data reflect the values of the adjusted informational energy, which assess their degree of concentration.

The following equation reflects the model of informational energy:

$$
E_{i a j}=2 f_{1}^{2}+2 f_{2}^{2}-1
$$

where: $E_{i j}=$ adjusted informational energy; $f_{1}=$ the structure of incomes achieved by the selfemployed person from freelancing; $f_{2}=$ the structure of incomes achieved by the self-employed person from entrepreneurship $f_{1}=\xi$ and $f_{2}=\omega$. 
Table 1. Wealth of statistical information concerning the incomes achieved by a freelancer turned entrepreneur, in the period 2011-2017

\begin{tabular}{|c|c|c|c|}
\hline \multirow{2}{*}{ years } & \multicolumn{3}{|c|}{ incomes achieved by a self-employed person } \\
\cline { 2 - 4 } & $\begin{array}{c}\text { incomes from freelancing } \\
\text { (EUR) }\end{array}$ & $\begin{array}{c}\text { incomes from entrepreneurship } \\
\text { (EUR) }\end{array}$ & total incomes (EUR) \\
\hline 2011 & 10940 & 27736 & 38676 \\
\hline 2012 & 9172 & 29828 & 39000 \\
\hline 2013 & 9465 & 31472 & 40937 \\
\hline 2014 & 8927 & 36206 & 45133 \\
\hline 2015 & 7826 & 34930 & 42756 \\
\hline 2016 & 5829 & 41048 & 46877 \\
\hline 2017 & 6294 & 39622 & 45916 \\
\hline
\end{tabular}

Source: Incomes of freelancer turned entrepreneur from Romania.

The effects of these symbols make the adjusted informational energy to have the following shape:

$$
E_{i a j}=2 \xi^{2}+2 \omega^{2}-1=\phi(\xi, \omega),
$$

The values of the structures of the incomes from freelancing, respectively the incomes from entrepreneurship, in the case of a self-employed person, in the period 2011-2017, and the values of the adjusted informational energy, are highlighted in Table 2:

Table 2. The structures of incomes from freelancing, respectively the weights of incomes from entrepreneurship, in the case of a self-employed person, and the «boom» of the development of the adjusted informational energy, in the period 2011-2017

\begin{tabular}{|c|c|c|c|c|c|c|c|c|}
\hline \multirow{3}{*}{ 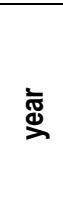 } & \multicolumn{4}{|c|}{ the structures of incomes } & \multirow{3}{*}{$f_{1}^{2}$} & \multirow{3}{*}{$f_{2}^{2}$} & \multirow{3}{*}{$\begin{array}{c}\text { informational } \\
\text { energy } \\
E_{i}=\sum_{i=1}^{2} f_{i}^{2}\end{array}$} & \multirow{3}{*}{$\begin{array}{c}\text { adjusted } \\
\text { informational } \\
\text { energy }\end{array}$} \\
\hline & \multicolumn{2}{|c|}{$\begin{array}{l}\text { the structures for } \\
\text { the incomes from } \\
\text { freelancing }\left(f_{1}\right)\end{array}$} & \multicolumn{2}{|c|}{$\begin{array}{l}\text { the structures for } \\
\text { the incomes from } \\
\text { entrepreneurship } \\
\left(f_{2}\right)\end{array}$} & & & & \\
\hline & & $(\%)$ & & $(\%)$ & & & & \\
\hline 2011 & 0,2829 & 28,29 & 0,7171 & 71,71 & 0,08003241 & 0,51423241 & 0,59426482 & 0,18852964 \\
\hline 2012 & 0,2352 & 23,52 & 0,7648 & 76,48 & 0,05531904 & 0,58491904 & 0,64023808 & 0,28047616 \\
\hline 2013 & 0,2312 & 23,12 & 0,7688 & 76,88 & 0,05345344 & 0,59105344 & 0,64450688 & 0,28901376 \\
\hline 2014 & 0,1978 & 19,78 & 0,8022 & 80,22 & 0,03912484 & 0,64352484 & 0,68264968 & 0,36 \\
\hline 2015 & 0,1830 & 18,30 & 0,8170 & 81,70 & 0,033489 & 0,667489 & 0,700978 & 956 \\
\hline 2016 & 0,1243 & 12,43 & 0,8757 & 87,57 & 0,01545049 & 0,76685049 & 0,78230098 & 0,56460196 \\
\hline 2017 & 0,1371 & 13,71 & 0,8629 & 86,29 & 0,01879641 & 0,74459641 & 0,76339282 & 0,52678564 \\
\hline
\end{tabular}
(2011)

note: $\xi(1)$ and $\omega(1)$ highlight the current year $(2017) ; \xi(0)$ and $\omega(0)$ project the base year

$$
\xi(0)=0,2829 ; \omega(0)=0,7171 ; \xi(1)=0,1371 ; \omega(1)=0,8629
$$

Source: author's calculations.

Onicescu (1966) is the first researcher who introduced the concept of informational energy in an attempt to define a finer measure of dispersion distribution than Shannon's information entropy. Onicescu shows that information entropy increases when informational energy decreases. Based on the information importance, information asymmetry as an unequal distribution of information between at least two stakeholders about the various parameters processes and events in the innovation system can 
determine problems (Omelianenko, 2017).

Results. The focalization of $\xi$ factor. If the projection of the mathematical modelling for $\xi$ variable, where $\xi=$ incomes from freelancing achieved by the self-employed person, follows a linear trend $\xi_{t_{i}}=$ $=a+b \cdot t_{i}$, then $a$ and $b$ parameters will be:

$$
\begin{aligned}
& \left\{\begin{array}{l}
n \cdot a=\sum_{i=-m}^{m} \xi_{i} \\
b \cdot \sum_{i=-m}^{m} t_{i}^{2}=\sum_{i=-m}^{m} t_{i} \cdot \xi_{i}
\end{array}\right. \\
& a=\frac{\sum_{i=-m}^{m} \xi_{i}}{n} \text { and } b=\frac{\sum_{i=-m}^{m} t_{i} \cdot \xi_{i}}{\sum_{i=-m}^{m} t_{i}^{2}}
\end{aligned}
$$

Table 3. Wealth of statistical information's for $\xi$ variable, if the projection, concerning the incomes from freelancing achieved by a self-employed person, displays a linear trend

\begin{tabular}{|c|c|c|c|c|c|c|}
\hline \multirow{2}{*}{ years } & \multirow{2}{*}{$\begin{array}{c}\text { the incomes from } \\
\text { freelancing (EUR) } \\
\end{array}$} & $\xi_{i}$ & \multicolumn{5}{|c|}{ linear trend } \\
\cline { 3 - 7 } & $\mathrm{t}_{\mathrm{i}}$ & $\mathrm{t}_{\mathrm{i}}^{2}$ & $\mathrm{t}_{\mathrm{i}} \xi_{\mathrm{i}}$ & $\xi_{\mathrm{t}_{\mathrm{i}}}=\mathrm{a}+\mathrm{bt}_{\mathrm{i}}$ & $\left|\xi_{\mathrm{i}}-\xi_{\mathrm{t}_{\mathrm{i}}}\right|$ \\
\hline 2011 & 10.940 & -3 & 9 & -32820 & 10735,75 & 204,25 \\
\hline 2012 & 9.172 & -2 & 4 & -18344 & 9940,642857 & 768,64 \\
\hline 2013 & 9.465 & -1 & 1 & -9465 & 9145,535714 & 319,46 \\
\hline 2014 & 8.927 & 0 & 0 & 0 & 8350,428571 & 576,57 \\
\hline 2015 & 7.826 & +1 & 1 & 7826 & 7555,321427 & 270,68 \\
\hline 2016 & 5.829 & +2 & 4 & 11658 & 6760,214285 & 931,21 \\
\hline 2017 & 6.294 & +3 & 9 & 18882 & 5965,107142 & 328,89 \\
\hline TOTAL & 58453 & & 28 & -22263 & 58453 & 3399,7 \\
\hline
\end{tabular}

Source: author's calculations.

$$
\begin{gathered}
a=\frac{58453}{7}=8350,428571 \text { and } b=\frac{-22263}{28}=-795,1071429 \\
v_{I}=\left[\frac{\sum_{i=-m}^{m}\left|\xi_{i}-\xi_{t_{i}}^{I}\right|}{n}: \frac{\sum_{i=-m}^{m} \xi_{i}}{n}\right] \cdot 100=\frac{\sum_{i=-m}^{m}\left|\xi_{i}-\xi_{t_{i}}^{I}\right|}{\sum_{i=-m}^{m} \xi_{i}} \cdot 100=\frac{3399,7}{58453} \cdot 100=5,816125 \%
\end{gathered}
$$

If the projection of the mathematical modelling for $\xi$ variable, where $\xi=$ incomes from freelancing amassed by the self-employed person, configures a quadratic trend, then $a$ and $b$ parameters will be:

$$
\begin{gathered}
\left\{\begin{array}{l}
n \cdot a+c \sum_{i=-m}^{m} t_{i}^{2}=\sum_{i=-m}^{m} \xi_{i} \\
b \cdot \sum_{i=-m}^{m} t_{i}^{2}=\sum_{i=-m}^{m} t_{i} \cdot \xi_{i} \\
a \cdot \sum_{i=-m}^{m} t_{i}^{2}+c \sum_{i=-m}^{m} t_{i}^{4}=\sum_{i=-m}^{m} t_{i}^{2} \cdot \xi_{i}
\end{array}\right. \\
a=\frac{\sum_{i=-m}^{m} t_{i}^{4} \cdot \sum_{i=-m}^{m} \xi_{i}-\sum_{i=-m}^{m} t_{i}^{2} \cdot \sum_{i=-m}^{m} t_{i}^{2} \cdot \xi_{i}}{n \cdot \sum_{i=-m}^{m} t_{i}^{4}-\left(\sum_{i=-m}^{m} t_{i}^{2}\right)^{2}} \\
b=\frac{\sum_{i=-m}^{m} t_{i} \cdot \xi_{i}}{\sum_{i=-m}^{m} t_{i}^{2}} \\
\quad c=\frac{n \cdot \sum_{i=-m}^{m} t_{i}^{2} \cdot \xi_{i}-\sum_{i=-m}^{m} t_{i}^{2} \cdot \sum_{i=-m}^{m} \xi_{i}}{n \cdot \Sigma_{i=-m}^{m} t_{i}^{4}-\left(\sum_{i=-m}^{m} t_{i}^{2}\right)^{2}} \\
a=\frac{196 \cdot 58453-28 \cdot 232401}{7 \cdot 196-(28)^{2}}=8417,619048 ; \\
b=\frac{-22263}{28}=-795,1071429
\end{gathered}
$$




$$
\begin{gathered}
c=\frac{7 \cdot 232401-28 \cdot 58453}{7 \cdot 196-(28)^{2}}=-16,79761905 \\
v_{I I}=\left[\frac{\sum_{i=-m}^{m}\left|\xi_{i}-\xi_{i}^{I I}\right|}{n}: \frac{\sum_{i=-m}^{m} \xi_{i}}{n}\right] \cdot 100=\frac{\sum_{i=-m}^{m}\left|\xi_{i}-\xi_{t_{i}}^{I I}\right|}{\sum_{i=-m}^{m} \xi_{i}} \cdot 100=\frac{3399,71}{58453} \cdot 100=5,816142 \%
\end{gathered}
$$

Table 4. Wealth of statistical information's for $\xi$ variable, if the projection, concerning the incomes from freelancing achieved by the self-employed person, displays a quadratic trend

\begin{tabular}{|c|c|c|c|c|c|c|}
\hline \multirow{2}{*}{ Years } & \begin{tabular}{c} 
The incomes \\
from freelancing \\
(EUR) \\
\cline { 3 - 7 }
\end{tabular} & $\xi_{i}^{2}$ & $\mathrm{t}_{\mathrm{i}}^{4}$ & $\mathrm{t}_{\mathrm{i}}^{2} \cdot \xi_{\mathrm{i}}$ & $\xi_{\mathrm{t}_{\mathrm{i}}}=\mathrm{a}+\mathrm{bt}_{\mathrm{i}}+\mathrm{ct}_{\mathrm{i}}^{2}$ & $\left|\xi_{\mathrm{i}}-\xi_{\mathrm{t}_{\mathrm{i}} \mid}\right|$ \\
\hline 2011 & 10.940 & 9 & 81 & 98460 & 10651,76191 & 286,24 \\
\hline 2012 & 9.172 & 4 & 16 & 36688 & 9940,642858 & 768,64 \\
\hline 2013 & 9.465 & 1 & 1 & 9465 & 9195,928572 & 269,07 \\
\hline 2014 & 8.927 & 0 & 0 & 0 & 8417,619048 & 509,38 \\
\hline 2015 & 7.826 & 1 & 1 & 7826 & 7605,714286 & 220,29 \\
\hline 2016 & 5.829 & 4 & 16 & 23316 & 6760,214286 & 931,21 \\
\hline 2017 & 6.294 & 9 & 81 & 56646 & 5881,119048 & 412,88 \\
\hline Total & 58453 & 28 & 196 & 232401 & 58453 & 3399,71 \\
\hline
\end{tabular}

Source: author's calculations.

If the projection of the mathematical modelling for $\xi$ variable, where $\xi=$ incomes from freelancing highlighted by the self-employed person, describes an exponential trend $\xi_{t_{i}}=a b^{t_{i}}$, then $a$ and $b$ parameters will be:

$$
\begin{array}{r}
\left\{\begin{array}{l}
n \cdot \lg a=\sum_{i=-m}^{m} \lg \xi_{i} \\
\lg b \cdot \sum_{i=-m}^{m} t_{i}^{2}=\sum_{i=-m}^{m} t_{i} \cdot \lg \xi_{i}
\end{array}\right. \\
\lg a=\frac{\sum_{i=-m}^{m} \lg \xi_{i}}{n} \text { and } \lg b=\frac{\sum_{i=-m}^{m} t_{i} \cdot \lg \xi_{i}}{\sum_{i=-m}^{m} t_{i}^{2}}
\end{array}
$$

\begin{tabular}{|c|c|c|c|c|c|c|c|}
\hline \multirow[b]{2}{*}{ Years } & \multirow{2}{*}{$\begin{array}{c}\text { The incomes } \\
\text { from freelancing } \\
\text { (EUR) } \\
\xi_{i}\end{array}$} & \multicolumn{6}{|c|}{ Exponential trend } \\
\hline & & $\lg \xi_{i}$ & $t_{i}^{2}$ & $t_{i} \lg \xi_{i}$ & $\lg \xi_{t_{i}}=\lg a+t_{i} \cdot \lg b$ & $\xi_{t_{i}}=a b^{t_{i}}$ & $\left|\xi_{i}-\xi_{t_{i}}\right|$ \\
\hline 2011 & 10.940 & 4,039017322 & 9 & $-12,11705197$ & 4,04053837 & 10978,3828 & 38,38 \\
\hline 2012 & 9.172 & 3,962464046 & 4 & $-7,924928092$ & 3,997805016 & 9949,586132 & 777,59 \\
\hline 2013 & 9.465 & 3,976120618 & 1 & $-3,976120618$ & 3,955071662 & 9017,199162 & 447,8 \\
\hline 2014 & 8.927 & 3,950705535 & 0 & 0 & 3,912338308 & 8172,187229 & 754,81 \\
\hline 2015 & 7.826 & 3,893539844 & 1 & $+3,89359844$ & 3,869604954 & 7406,362321 & 419,64 \\
\hline 2016 & 5.829 & 3,765594055 & 4 & $+7,531188111$ & 3,8268716 & 6712,30373 & 883,3 \\
\hline 2017 & 6.294 & 3,798926739 & 9 & $+11,39678022$ & 3,784138246 & 6083,286156 & 210,71 \\
\hline Total & 58453 & & 28 & $-1,196533913$ & & & 3532,23 \\
\hline
\end{tabular}

Table 5. Wealth of statistical information for $\xi$ variable, if the projection, regarding the incomes from freelancing achieved by the self-employed person, displays an exponential trend

Source: author's calculations. 


$$
\begin{gathered}
\lg a=\frac{27,38636816}{7}=3,912338308 ; \lg b=\frac{-1,19533913}{28}=-0,042733354 \\
v_{\text {exp }}=\left[\frac{\sum_{i=-m}^{m}\left|\xi_{\mathrm{i}}-\xi_{i_{\mathrm{i}}}^{\exp }\right|}{n}: \frac{\sum_{i=-m}^{\mathrm{m}} \xi_{\mathrm{i}}}{n}\right] \cdot 100=\frac{\sum_{i=-m}^{m}\left|\xi_{\mathrm{i}}-\xi_{i_{\mathrm{i}}}^{\exp }\right|}{\sum_{i=-m}^{m} \xi_{\mathrm{i}}} \cdot 100=\frac{3532,23}{58453} \cdot 100=6,042854 \% \\
v_{I}=5,816125 \%<v_{I I}=5,816142 \%<v_{\text {exp }}
\end{gathered}
$$

The modelling of the projection concerning $\xi$ factor, which focuses the incomes from freelancing achieved by self-employed person, follows a linear leaning of shape $\xi_{t_{i}}=a+b t_{t i}$.

The focalization of $\omega$ factor. If the projection of the mathematical modelling for $\omega$ variable, where $\omega=$ incomes from entrepreneurship amassed by self-employed person, sketches a linear trend $\omega_{t}=a+b \cdot t_{i}$, then $a$ and $b$ parametres will be:

$$
a=\frac{\sum_{i=-m}^{m} \omega_{i}}{n} \text { and } b=\frac{\sum_{i=-m}^{m} t_{i^{\prime}} \omega_{i}}{\sum_{i=-m}^{m} t_{i}^{2}}
$$

Table 6. Wealth of statistical informations for $\omega$ variable, if the projection, regarding the incomes from entrepreneurship amassed by self-employed person, displays a linear trend

\begin{tabular}{|c|c|c|c|c|c|c|}
\hline \multirow{2}{*}{ years } & \multirow{2}{*}{$\begin{array}{c}\text { incomes from } \\
\text { entrepreneurship (EUR) } \\
\omega_{i}\end{array}$} & \multicolumn{5}{|c|}{ linear trend } \\
\cline { 3 - 7 } & 27.736 & $\mathrm{t}_{\mathrm{i}}$ & $\mathrm{t}_{\mathrm{i}}^{2}$ & $\mathrm{t}_{\mathrm{i}} \omega_{\mathrm{i}}$ & $\omega_{\mathrm{t}_{\mathrm{i}}}=\mathrm{a}+\mathrm{bt}_{\mathrm{i}}$ & $\left|\omega_{\mathrm{i}}-\omega_{\mathrm{t}_{\mathrm{i}}}\right|$ \\
\hline 2011 & 29.828 & -3 & 9 & -83208 & 27810,71429 & 74,71 \\
\hline 2012 & 31.472 & -1 & 1 & -31472 & 32207,57143 & 735,57 \\
\hline 2013 & 36.206 & 0 & 0 & 0 & 34406 & 1800 \\
\hline 2014 & 34.930 & +1 & 1 & +34930 & 36604,42857 & 1674,43 \\
\hline 2015 & 41.048 & +2 & 4 & +82096 & 38802,85714 & 2245,14 \\
\hline 2016 & 39.622 & +3 & 9 & +118866 & 41001,28571 & 1379,29 \\
\hline 2017 & 240842 & & 28 & 61556 & 240842 & 5331,7 \\
\hline Total & & & & & \\
\hline
\end{tabular}

Source: author's calculations.

$$
\begin{gathered}
a=\frac{240842}{7}=34406 \text { and } b=\frac{61556}{28}=2198,428571 \\
v_{I}=\left[\frac{\sum_{i=-m}^{m}\left|\omega_{i}-\omega_{t_{i}}^{t}\right|}{n}: \frac{\sum_{i=-m}^{m} \omega_{i}}{n}\right] \cdot 100=\frac{\sum_{i=-m}^{m}\left|\omega_{i}-\omega_{t_{i}}^{I}\right|}{\sum_{i=-m}^{m} \omega_{i}} \cdot 100=\frac{5331,7}{240842} \cdot 100=2,21 \%
\end{gathered}
$$

If the projection of the mathematical modelling for $\omega$ variable, where $\omega=$ incomes from entrepreneurship achieved by the self-employed person, configures a quadratic trend $\omega_{t_{i}}=a+b$. $t_{i}+c t_{i}^{2}$, then $a$ and $b$ parametres will be.

$$
\begin{gathered}
a=\frac{\sum_{i=-m}^{m} t_{i}^{4} \cdot \sum_{i=-m}^{m} \omega_{i}-\sum_{i=-m}^{m} t_{i}^{2} \cdot \sum_{i=-m}^{m} t_{i}^{2} \cdot \omega_{i}}{n \cdot \sum_{i=-m}^{m} t_{i}^{4}-\left(\sum_{i=-m}^{m} t_{i}^{2}\right)^{2}} \\
b=\frac{\sum_{i=-m}^{m} t_{i} \cdot \omega_{i}}{\sum_{i=-m}^{m} t_{i}^{2}} \\
c=\frac{n \cdot \sum_{i=-m}^{m} t_{i}^{2} \cdot \omega_{i}-\sum_{i=-m}^{m} t_{i}^{2} \cdot \sum_{i=-m}^{m} \omega_{i}}{n \cdot \sum_{i=-m}^{m} t_{i}^{4}-\left(\sum_{i=-m}^{m} t_{i}^{2}\right)^{2}}
\end{gathered}
$$




$$
\begin{gathered}
a=\frac{196 \cdot 240842-28 \cdot 956128}{7 \cdot 196-(28)^{2}}=34750,7619 ; \\
b=\frac{61556}{28}=2198,428571 \\
c=\frac{7 \cdot 956128-28 \cdot 240842}{7 \cdot 196-(28)^{2}}=86,19047619 \\
v_{I I}=\left[\frac{\sum_{i=-m}^{m}\left|\omega_{i}-\omega_{t_{i}}^{I I}\right|}{n}: \frac{\sum_{i=-m}^{m} \omega_{i}}{n}\right] \cdot 100=\frac{\sum_{i=-m}^{m}\left|\omega_{i}-\omega_{t_{i}}^{I I}\right|}{\sum_{i=-m}^{m} \omega_{i}} \cdot 100=\frac{8113,22}{240842} \cdot 100=3,37 \%
\end{gathered}
$$

\begin{tabular}{|c|c|c|c|c|c|c|}
\hline \multirow[b]{2}{*}{ years } & \multirow{2}{*}{$\begin{array}{l}\text { incomes from entrepreneurship } \\
\text { (EUR) } \\
\omega_{i}\end{array}$} & \multicolumn{5}{|c|}{ parabolic trend } \\
\hline & & $t_{i}^{2}$ & $t_{i}^{4}$ & $t_{i}^{2} \cdot w_{i}$ & $\omega_{t_{i}}=a+b t_{i}+c t_{i}^{2}$ & $\left|\omega_{i}-\omega_{t_{i}}\right|$ \\
\hline 2011 & 27.736 & 9 & 81 & 249624 & 27379,7619 & 356,24 \\
\hline 2012 & 29.828 & 4 & 16 & 119312 & 30009,14285 & 181,14 \\
\hline 2013 & 31.472 & 1 & 1 & 31472 & 32466,14285 & 994,14 \\
\hline 2014 & 36.206 & 0 & 0 & 0 & 34750,7619 & 1455,25 \\
\hline 2015 & 34.930 & 1 & 1 & 34930 & 36862,99999 & 1932,99 \\
\hline 2016 & 41.048 & 4 & 16 & 164192 & 38802,85714 & 2245,15 \\
\hline 2017 & 39.622 & 9 & 81 & 356598 & 40570,33333 & 948,33 \\
\hline total & 240842 & 28 & 196 & 956128 & 240842 & 8113,22 \\
\hline
\end{tabular}

Table 7. Wealth of statistical information's for $\omega$ variable, if the projection, regarding the incomes from entrepreneurship highlighted by self-employed person, displays a quadratic trend

Source: author's calculations.

If the projection of the mathematical 334odelling for $\omega$ variable, where $\omega=$ incomes from entrepreneurship highlighted by self-employed person, describes an exponential trend $\omega_{t_{i}}=a b^{t_{i}}$, then $a$ and $b$ parametres will be:

$$
\lg a=\frac{\sum_{i=-m}^{m} \lg \omega_{i}}{n} \text { and } \lg b=\frac{\sum_{i=-m}^{m} t_{i} \cdot \lg \omega_{i}}{\sum_{i=-m}^{m} t_{i}^{2}}
$$

\begin{tabular}{|c|c|c|c|c|c|c|c|}
\hline \multirow[b]{2}{*}{ years } & \multirow{2}{*}{$\begin{array}{c}\text { incomes from } \\
\text { entrepreneurship } \\
\text { (EUR) } \\
\omega_{i}\end{array}$} & \multicolumn{6}{|c|}{ exponential trend } \\
\hline & & $\lg \omega_{\mathrm{i}}$ & $t_{i}^{2}$ & $t_{i} \lg \omega_{i}$ & $\lg \omega_{t_{i}}=\lg a+t_{i} \cdot \lg b$ & $\omega_{t_{i}}=a b^{t_{i}}$ & $\left|\omega_{i}-\omega_{t_{i}}\right|$ \\
\hline 2011 & 27.736 & 4,443043829 & 9 & $-13,32913149$ & 4,448333443 & 28075,88423 & 339,88 \\
\hline 2012 & 29.828 & 4,474624134 & 4 & $-8,949248269$ & 4,476450859 & 29953,72643 & 125,73 \\
\hline 2013 & 31.472 & 4,497924343 & 1 & $-4,497924343$ & 4,504568275 & 31957,16721 & 485,17 \\
\hline 2014 & 36.206 & 4,558780547 & 0 & 0 & 4,532685691 & 34094,60717 & 2111,39 \\
\hline 2015 & 34.930 & 4,543198586 & 1 & 4,543198586 & 4,560803107 & 36375,00879 & 1445,01 \\
\hline 2016 & 41.048 & 4,613292006 & 4 & 9,226584003 & 4,588920523 & 38807,934 & 2240,07 \\
\hline 2017 & 39.622 & 4,597936394 & 9 & 13,79380918 & 4,617037941 & 41403,58444 & 1781,58 \\
\hline Total & 240842 & 31,72879982 & 28 & 0,787287667 & & & 8528,83 \\
\hline
\end{tabular}

Table 8. Wealth of statistical information's for $\omega$ variable, if the projection, regarding the incomes from entrepreneurship achieved by the self-employed person, displays an exponential trend 


$$
\begin{gathered}
\lg a=\frac{31,72879982}{7}=4,532685691 \text { and } \lg b=\frac{0,787287667}{28}=0,028117416 \\
v_{\text {exp }}=\left[\frac{\sum_{i=-m}^{m \Sigma}\left|\omega_{i}-\omega_{t_{i}}^{e x p}\right|}{n}: \frac{\sum_{i=-m}^{m} \omega_{i}}{n}\right] \cdot 100=\frac{\sum_{i=-m}^{m \Sigma}\left|\omega_{i}-\omega_{t_{i}}^{e x p}\right|}{\sum_{i=-m}^{m} \omega_{i}} \cdot 100=\frac{8528,83}{3,854} \cdot 100=3,54 \% \\
v_{I}=2,21 \%<v_{I I}=3,37 \%<v_{\text {exp }}
\end{gathered}
$$

The modeling of the projection concerning wfactor, which focuses the incomes from entrepreneurship amassed by a Self-employed person, follows a linear leaning of shape

$$
\omega_{t_{i}}=a+b t_{t i}
$$

The influences of the structures concerning the incomes achieved by a self-employed person on informational energy dynamics.

The influence in relative sizes, regarding the structures of incomes from freelancing achieved by a Self-employed person, on adjusted informational energy dynamics, in 2017 comparable to 2011, can be expressed as follows:

$$
I_{1 / 0}^{\phi(\xi / \omega)}=e^{\int_{\left(P_{0} P_{1}\right)} \frac{4 \xi}{2 \xi^{2}+2 \omega^{2}-1} d \xi}
$$

The influence in relative sizes, concerning the weights of incomes from entrepreneurship achieved by a Self-employed person, on adjusted informational energy dynamics, in 2017 comparable to 2011, is:

$$
I_{1 / 0}^{\phi(\omega / \xi)}=e^{\int_{\left(P_{0} P_{1}\right)} \frac{4 \omega}{2 \xi^{2}+2 \omega^{2}-1} d \omega}
$$

$\xi$ factor (the structure of incomes from freelancing achieved by a self-employed person) reflects a linear function $\xi_{\mathrm{t}_{\mathrm{i}}}=\mathrm{a}+\mathrm{bt}_{\mathrm{ti}}$. The focalization of the geometrical decomposition concerning the adjusted informational energy dynamics, in 2017 comparable to 2011, under the influences represented by the structures of the incomes from freelancing, respectively the weights of the incomes from entrepreneurship, highlighted by a self-employed person, outlines the following configuration of the conditions:

$$
\begin{gathered}
\xi(0)=a \text { şi } \xi(1)=a+b=\xi(0)+b \Rightarrow b=\xi(1)-\xi(0)=\Delta_{1 / 0}^{\xi} \\
\xi=\xi(0)+\Delta_{1 / 0}^{\xi} \cdot t \Rightarrow d \xi=\Delta_{1 / 0}^{\xi} \cdot d t
\end{gathered}
$$

The following integral is computed, considering that $\xi+\omega=1$ or $100 \%$ :

$$
\begin{aligned}
& \int_{\left(P_{0} P_{1}\right)} \frac{4 \xi}{2 \xi^{2}+2 \omega^{2}-1} d x=\int_{\xi(0)}^{\xi(1)} \frac{4 \xi}{(2 \xi-1)^{2}} d \xi=4 \int_{0}^{1} \frac{\left[\xi(0)+\Delta_{1 / 0}^{\xi} \cdot t\right] \cdot \Delta_{1 / 0}^{\xi}}{\left[2 \xi(0)+2 \Delta_{1 / 0}^{\xi} \cdot t-1\right]^{2}} d t= \\
= & 4 \xi(0) \cdot \Delta_{1 / 0}^{\xi} \int_{0}^{1} \frac{d t}{\left[2 \Delta_{1 / 0}^{\xi} \cdot t+2 \xi(0)-1\right]^{2}}+4\left(\Delta_{1 / 0}^{\xi}\right)^{2} \int_{0}^{1} \frac{t}{\left[2 \Delta_{1 / 0}^{\xi} \cdot t+2 \xi(0)-1\right]^{2}} d t=
\end{aligned}
$$




$$
\begin{aligned}
& =4 \xi(0) \cdot \Delta_{\frac{1}{0}}^{\xi} \int_{0}^{1} \frac{d t}{4\left(\frac{\Delta_{\frac{1}{0}}^{\xi}}{)^{2}}\left[t+\frac{2 \xi(0)-1}{2 \Delta_{\frac{1}{0}}^{\xi}}\right]^{2}\right.}+4\left(\Delta_{\frac{1}{0}}^{\xi}\right)^{2} \int_{0}^{1} \frac{t}{4\left(\Delta_{\frac{1}{0}}^{\xi}\right)^{2} \cdot t^{2}+4[2 \xi(0)-1] \cdot \Delta_{\frac{1}{0}}^{\xi} \cdot t+[2 \xi(0)-1]^{2}} d t= \\
& =\frac{\xi(0)}{\Delta_{1 / 0}^{\xi}} \int_{0}^{1} \frac{d t}{\left[t+\frac{2 \xi(0)-1}{2 \Delta_{1 / 0}^{\xi}}\right]^{2}}+\frac{1}{2} \int_{0}^{1} \frac{4\left(\Delta_{1 / 0}^{\xi}\right)^{2} \cdot 2 t+4[2 \xi(0)-1] \cdot \Delta_{1 / 0}^{x}}{4\left(\Delta_{1 / 0}^{\xi}\right)^{2} t^{2}+4[2 \xi(0)-1] \cdot \Delta_{1 / 0}^{\xi} \cdot t+[2 \xi(0)-1]^{2}} d t- \\
& -\frac{1}{2} \int_{0}^{1} \frac{4[2 \xi(0)-1] \cdot \Delta_{1 / 0}^{\xi}}{\left[2 \Delta_{1 / 0}^{\xi} \cdot t+2 \xi(0)-1\right]^{2}} d t=\frac{\xi(0)}{\Delta_{\frac{1}{0}}^{\xi}}\left[-\frac{1}{t+\frac{2 \xi(0)-1}{2 \Delta_{\frac{1}{0}}^{\xi}}}\right]+\frac{1}{\frac{1}{0}} \ln \left[4\left(\Delta_{\frac{1}{0}}^{\xi}\right)^{2} t^{2}+4[2 \xi(0)-1] \cdot \Delta_{\frac{1}{0}}^{\xi} \cdot t+\right. \\
& \left.+[2 \xi(0)-1]^{2}\right]-2[2 \xi(0)--1] \Delta_{\frac{1}{0}}^{\xi} \int_{0}^{1} \frac{d t}{4\left(\frac{\left.\Delta_{\frac{1}{0}}^{\xi}\right)^{2}\left[t+\frac{2 \xi(0)-1}{2 \Delta_{\frac{1}{0}}^{\xi}}\right]^{2}}{\frac{1}{0}}\right]^{2}}=-\frac{\xi(0)}{\Delta_{\frac{1}{0}}^{\xi}}\left[\frac{1}{1+\frac{2 \xi(0)-1}{2 \Delta_{\frac{1}{0}}^{\xi}}}-\frac{1}{\frac{2 \xi(0)-1}{2 \Delta_{\frac{1}{0}}^{\xi}}}\right]+ \\
& +\frac{1}{2} \ln \left[\frac{2 \Delta_{\frac{1}{0}}^{\xi}+[2 \xi(0)-1]}{2 \xi(0)-1}\right]^{2}--\frac{2 \xi(0)-1}{2 \Delta_{\frac{1}{0}}^{\xi}}\left[-\frac{1}{t+\frac{2 \xi(0)-1}{2 \Delta_{\frac{1}{0}}^{\xi}}}\right]=\frac{4 \xi(0) \Delta_{\frac{1}{0}}^{\xi}}{[2 \xi(1)-1][2 \xi(0)-1]}+\ln \frac{2 \xi(1)-1}{2 \xi(0)-1}+ \\
& +\frac{2 \xi(0)-1}{2 \Delta_{\frac{1}{0}}^{\xi}}\left[\frac{1}{1+\frac{2 \xi(0)-1}{2 \Delta_{\frac{1}{\overline{0}}}^{\xi}}}-\frac{1}{\frac{2 \xi(0)-1}{2 \Delta_{\frac{1}{\overline{0}}}^{\xi}}}\right]=\frac{4 \xi(0) \cdot \Delta_{\frac{1}{0}}^{\xi}}{[2 \xi(1)-1][2 \xi(0)-1]}+\ln \frac{2 \xi(1)-1}{2 \xi(0)-1}-\frac{2[2 \xi(0)-1] \cdot \Delta_{\frac{1}{0}}^{\xi}}{[2 \xi(1)-1][2 \xi(0)-1]}= \\
& =\frac{2 \Delta_{\frac{1}{0}}^{\xi}}{[2 \xi(1)-1][2 \xi(0)-1]}+\ln \frac{2 \xi(1)-1}{2 \xi(0)-1}=\frac{2(0,1371-0,2829)}{(2 \cdot 0,1371-1)(2 \cdot 0,2829-1)}+\ln \frac{2 \cdot 0,1371-1}{2 \cdot 0,2829-1}= \\
& =-0,411526872 \\
& I_{1 / 0}^{\phi(\xi / \omega)}=e^{\int_{\left(P_{0} P_{1}\right)} \frac{4 \xi}{2 \xi^{2}+2 \omega^{2}-1} d \xi}=e^{-0,411526872}=0,662637714 \text { or } 66,26 \%
\end{aligned}
$$

The adjusted informational energy decreased in relative sizes, in 2017 comparable to 2011, under the focalization of the influence regarding the structures of the incomes amassed by a Self-employed person from freelancing, with $33,74 \%$.

Moreover, $\omega$ factor (the structure of the incomes achieved by a Self-employed person from entrepreneurship), varies after a linear function $\omega_{t_{i}}=a+b t_{t i}$

$$
\begin{gathered}
\omega(0)=a \text { şi } \omega(1)=a+b=\omega(0)+b \Rightarrow b=\omega(1)-\omega(0)=\Delta_{1 / 0}^{\omega} \\
\omega=\omega(0)+\Delta_{1 / 0}^{\omega} \cdot t \Rightarrow d \omega=\Delta_{1 / 0}^{\omega} \cdot d t
\end{gathered}
$$

Analogous, the following integral needs to be computed, considering that $\xi+\omega=1$ or $100 \%$ :

$$
\int_{\left(P_{0} P_{1}\right)} \frac{4 \omega}{2 \xi^{2}+2 \omega^{2}-1} d \omega=\int_{\omega(0)}^{\omega(1)} \frac{4 \omega}{(2 \xi-1)^{2}} d \omega=4 \int_{0}^{1} \frac{\left[\omega(0)+\Delta_{1 / 0}^{\omega} \cdot t\right] \cdot \Delta_{1 / 0}^{\omega}}{\left[2 \xi(0)+2 \Delta_{1 / 0}^{\xi} \cdot t-1\right]^{2}} d t=
$$




$$
\begin{gathered}
=\frac{2 \Delta_{1 / 0}^{\omega}}{[2 \omega(1)-1][2 \omega(0)-1]}+\ln \frac{2 \omega(1)-1}{2 \omega(0)-1}= \\
=\frac{2(0,8629-0,7171)}{(2 \cdot 0,8629-1)(2 \cdot 0,7171-1)}+\ln \frac{2 \cdot 0,8629-1}{2 \cdot 0,7171-1}=1,439065347 \\
I_{1 / 0}^{\phi(\omega / \xi)}=e^{\int_{\left(P_{0} P_{1}\right)} \frac{4 \omega}{2 \xi^{2}+2 \omega^{2}-1} d \omega}=e^{1,439065347}=4,216752772 \text { or } 421,68 \%
\end{gathered}
$$

The adjusted informational energy increased in relative sizes, in 2017 face to 2011, under the incidence of the influence regarding the structures of the incomes amassed by a self-employed person from entrepreneurship, with $321,38 \%$.

In the focalization of the arithmetical decomposition, concerning $E_{i a j}$ indicator dynamics, through Factors Path Method, the values, in absolute sizes, of the influences regarding each factor, $\xi$ factor, respectively $\omega$ factor, are estimated:

$$
\begin{aligned}
\Delta_{1 / 0}^{\phi(\xi / \omega)} & =\int_{\left(P_{0} P_{1}\right)} \phi_{\xi}^{\prime} d \xi=4 \int_{\left(P_{0} P_{1}\right)} \xi d \xi \\
\text { and } \Delta_{1 / 0}^{\phi(\omega / \xi)} & =\int_{\left(P_{0} P_{1}\right)} \phi_{\omega}^{\prime} d \xi=4 \int_{\left(P_{0} P_{1}\right)} \omega d \omega
\end{aligned}
$$

Because the path of $\xi$ factor is a linear trend model, the decomposition is as follows:

$$
\begin{gathered}
\Delta_{1 / 0}^{\phi(\xi / \omega)}=\int_{\left(P_{0} P_{1}\right)} \phi_{\xi}^{\prime} d \xi=4 \int_{\left(P_{0} P_{1}\right)} \xi d \xi=4 \int_{\xi(0)}^{\xi(1)} \xi d \xi=4 \int_{0}^{1}\left[\xi(0)+\Delta_{1 / 0}^{\xi} \cdot t\right] \cdot \Delta_{\frac{1}{0}}^{\xi} d t= \\
=4 \xi(0) \cdot \Delta_{1 / 0}^{\xi} \int_{0}^{1} d t+4\left(\Delta_{1 / 0}^{\xi}\right)^{2} \int_{0}^{1} t d t==4 \xi(0) \cdot \Delta_{1 / 0}^{\xi} \cdot t+4\left(\Delta_{1 / 0}^{\xi}\right)^{2} \cdot \frac{t^{2}}{2}= \\
=4 \xi(0) \cdot \Delta_{1 / 0}^{\xi}+2\left(\Delta_{1 / 0}^{\xi}\right)^{2}=2 \cdot \Delta_{1 / 0}^{\xi} \cdot\left[2 \xi(0)+\Delta_{1 / 0}^{\xi}\right]= \\
=2 \cdot(0,1371-0,2829) \cdot[2 \cdot 0,2829+(0,1371-0,2829)]=-0,122472
\end{gathered}
$$

Also, because $\omega$ factor varies after a linear function, the same equation is highlighted:

$$
\begin{gathered}
\Delta_{1 / 0}^{\phi(\omega / \xi)}=\int_{\left(P_{0} P_{1}\right)} \phi_{\omega}^{\prime} d \omega=4 \int_{\left(P_{0} P_{1}\right)} \omega d \omega=4 \int_{\omega(0)}^{\omega(1)} \omega d \omega=4 \int_{0}^{1}\left[\omega(0)+\Delta_{1 / 0}^{\omega} \cdot t\right] \cdot \Delta_{\frac{1}{0}}^{\omega} d t= \\
=4 \omega(0) \cdot \Delta_{1 / 0}^{\omega} \int_{0}^{1} d t+4\left(\Delta_{1 / 0}^{\omega}\right)^{2} \int_{0}^{1} t d t=4 \omega(0) \cdot \Delta_{1 / 0}^{\omega} \cdot t+4\left(\Delta_{\frac{1}{0}}^{\omega}\right)^{2} \cdot \frac{t^{2}}{2}=4 \omega(0) \cdot \Delta_{\frac{1}{0}}^{\omega}+ \\
+2\left(\Delta_{\frac{1}{0}}^{\omega}\right)^{2}=2 \cdot \Delta_{\frac{1}{0}}^{\omega} \cdot\left[2 \omega(0)+\Delta_{\frac{1}{0}}^{\omega}\right]=2 \cdot(0,8629-0,7171) \cdot[2 \cdot 0,7171+ \\
+(0,8629-0,7171)]=0,460728
\end{gathered}
$$

The influences, in absolute sizes, regarding the weights of the incomes achieved by the selfemployed person from freelancing, respectively the weights of the incomes achieved by the selfemployed person from entrepreneurship, on adjusted informational energy dynamics, in 2017 comparable to 2011 , consisted in a subtraction with $-0,122472$, respectively a growth with 0,460728 .

Conclusions. This research is the first attempt in the entrepreneurship body of knowledge that analyses the distribution of incomes achieved by a freelancer turned entrepreneur through informational energy approach.

The index which reflects the dynamics concerning the level of the adjusted informational energy, under the influence of both factors, $\xi$ and $\omega$, respectively the structures of the incomes from freelancing 
and the weights of the incomes from entrepreneurship, achieved by the self-employed person, in 2017 comparable to 2011, is:

$$
I_{1 / 0}^{\phi(\xi \cup \omega)}=\frac{\left(E_{i a j}\right)_{2017}}{\left(E_{i a j}\right)_{2011}}=\frac{0,52678564}{0,18852964}=2,79417942 .
$$

The absolute variation of the adjusted informational energy, under the influence of both factors, $\xi$ and $\omega$, respectively the structures of the incomes from freelancing and the incomes from entrepreneurship, achieved by the freelancer turned entrepreneur, in 2017 comparable to 2011, is:

$$
\Delta_{1 / 0}^{\phi(\xi \cup \omega)}=\left(E_{i a j}\right)_{2017}-\left(E_{i a j}\right)_{2011}=0,52678564-0,18852964=0,338256 .
$$

The multiplication between the factorial influences has as effect the highlight of the general index:

$$
I_{1 / 0}^{\phi(\xi \cup \omega)}=I_{1 / 0}^{\phi(\xi / \omega)} \cdot I_{1 / 0}^{\phi(\omega / \xi)} \text { or } 2,79417942=0,66263772 * 4,216752772 .
$$

Moreover, the sum between the factorial influences has as «reflection» the general absolute increase:

$$
\Delta_{1 / 0}^{\phi(\xi \cup \omega)}=\Delta_{1 / 0}^{\phi(\xi / \omega)}+\Delta_{1 / 0}^{\phi(\omega / \xi)} \text { or } 0,338256=-0,122472+0,460728 .
$$

Findings highlight the growths concerning the values of the adjusted informational energy, as it follows: from to 0,18852964 in 2011 at 0,28047616 in 2012, at 0,28901376 in 2013, at 0,36529936 in 2014 , at 0,401956 in 2015 , at 0,56460196 in 2016 . These rises concerning the adjusted informational energy, which display the decrease of the entropy, are the effect of the growths regarding the incomes from entrepreneurship.

The maximum of the adjusted informational energy is in 2016 with the value of 0,56460196 and this «pole position» is equivalent with the magnification concerning the degree of concentration focused on structure in 2016. This positive value expresses the growth of the importance for the incomes from entrepreneurship.

In 2017, the adjusted informational energy subtracted at 0,52678564, value which reflects a negative phenomenon, a rise of the entropy as resultant of the increase concerning the incomes from freelancing, situation which drives at the diminution of the importance regarding the incomes from entrepreneurship.

No empirical studies exist in the field of entrepreneurship literature that focus on the analysis of freelancers turned entrepreneurs revenues, considering Onicescu's informational energy. Future research should consider a longitudinal research following this study's method with a larger sample of freelancers turned entrepreneurs. FsQCA can test the predictive validity of this conceptual model using a sample of freelancers turned entrepreneurs who will accept to share financial data (revenues from both activities) with the research team.

Author Contributions: G. O. contributed on conceptualization, methodology and formal analysis; D. D. contributed on the original draft preparation; A. C. contributed on writing-review and editing and supervision.

Funding. The paper is funded under the program of Minister of Science and Higher Education titled "Regional Initiative of Excellence" in 2019-2022, project number 018/RID/2018/19, the amount of funding PLN 10788423,16 


\section{References}

Amit, R., Glosten, L., \& Muller, E. (1993). Challenges to theory development in entrepreneurship research. Journal of Management studies, 30(5), 815-834.

Annink, A., Den Dulk, L., \& Amorós, J. E. (2016). Different strokes for different folks? The impact of heterogeneity in work characteristics and country contexts on work-life balance among the self-employed. International Journal of Entrepreneurial Behavior \& Research, 22(6), 880-902

Arthur, M. B., Claman, P. H., \& DeFillippi, R. J. (1995). Intelligent enterprise, intelligent careers.

Arthur, M., \& Rousseau, D. (1996). The Boundaryless Career, Oxford University Press. New York, NY.

Arulmani, G., Bakshi, A. J., Leong, F. T., \& Watts, A. G. (2014). Handbook of career development. International Perspectives. New York: Springer.

Autio, E. (2011). High-aspiration entrepreneurship. The Dynamics of Entrepreneurship: Evidence from Global Entrepreneurship Monitor Data, 251-275.

Backes-Gellner, U., \& Moog, P. (2013). The disposition to become an entrepreneur and the jacks-of-all-trades in social and human capital. The Journal of Socio-Economics, 47, 55-72.

Backes-Gellner, U., \& Moog, P. (2013). The disposition to become an entrepreneur and the jacks-of-all-trades in social and human capital. The Journal of Socio-Economics, 47, 55-72.

Bögenhold, D., Heinonen, J., \& Akola, E. (2014). Entrepreneurship and independent professionals: Social and economic logics. International Advances in Economic Research, 20(3), 295-310.

Borowiecki, R., \& Makieła, Z. J. (2019). Determinants of Entrepreneurship and Innovation Development in Local Areas of Economic Activity: a Case Study Analysis. In Forum Scientiae Oeconomia (Vol. 7, No. 2, pp. 7-24).

Burke, A., \& Van Stel, A. (2011). The entrepreneurship enabling role of freelancers: Theory with evidence from the construction industry. International Review of Entrepreneurship, 9(3), 131-158.

Damian, D., \& Manea, C. (2019). Causal recipes for turning fin-tech freelancers into smart entrepreneurs. Journal of Innovation \& Knowledge, https://doi.org/10.1016/j.jik.2019.01.003

Daum, K. (2005). Entrepreneurs: the artists of the business world. Journal of Business Strategy, 26(5), 53-57.

DeFillippi, R. J., \& Arthur, M. B. (2002). Career creativity to industry influence: A blueprint for the knowledge economy. Career Creativity. Explorations in the Remaking of Work, 298-313.

Falco, P., \& Haywood, L. (2016). Entrepreneurship versus joblessness: Explaining the rise in self-employment. Journal of Development Economics, 118, 245-265.

Florea, I. (1986). Statistics, Vol. I. Cluj-Napoca: University Babeş-Bolyai.

Greblikaite, J., Sroka, W., \& Grants, J. (2015). Development of social entrepreneurship in European Union: policy and situation of Lithuania and Poland. Transformations in Business \& Economics, 14.

Lichtarski, J., \& Trenkner, M. (2018). On the co-existence of innovation and creativity in the lean management environment. In Forum Scientiae Oeconomia (Vol. 6, №. 3, pp. 67-81).

McGrath, R. G., Mac Grath, R. G., \& MacMillan, I. C. (2000). The entrepreneurial mindset: Strategies for continuously creating opportunity in an age of uncertainty (Vol. 284). Harvard Business Press.

Omelianenko, V. A. (2017). Analysis of information asymmetry in innovation system security ensuring, Marketing and Management of Innovations, http://doi.org/10.21272/mmi.2017.4-17

Onicescu, O. (1966). The informational energy, component of the statistical barometer concerning the systems. Bucharest: Technical Publishing House.

Petrushenko, Y. M., \& Dudkin, O. V. (2014). Crowdfunding as an innovative tool for financing projects of social and economic development. Sumy: Marketing and Management of Innovations (in Ukr.).

Tams, S., \& Arthur, M. B. (2010). New directions for boundaryless careers: Agency and interdependence in a changing world. Journal of Organizational Behavior, 31(5), 629-646.

Unger, J. M., Rauch, A., Frese, M., \& Rosenbusch, N. (2011). Human capital and entrepreneurial success: A meta-analytical review. Journal of Business Venturing, 26(3), 341-358.

Van den Born, A., \& Van Witteloostuijn, A. (2013). Drivers of freelance career success. Journal of Organizational Behavior 34(1), 24-46.

Van den Born, A., \& Van Witteloostuijn, A. (2013). Drivers of freelance career success. Journal of Organizational Behavior, 34(1), 24-46

Wyrwich, M. (2013). Can socioeconomic heritage produce a lost generation with regard to entrepreneurship?. Journal of Business Venturing, 28(5), 667-682. 
Габріела Опайт, Ph.D., доцент Галатського університету "Дунареа де Джос» (Румунія);

Даніела Даміан, Університет "Дунареа де Джос»в Галаті (Румунія);

Олександру Капатіна, Ph.D., Університет «Дунареа де Джос» в Галаті (Румунія).

Фріланс як сучасна форма підприємницької діяльності: управління і розвиток професійної кар'єри

Підприємництво як форма ведення бізнесу набуває свого поширення серед фрілансерів у світі. 3 іншої точки зору, незначна кількість фрілансерів визнають свою професію як офіційну зайнятість, що є наслідком дії певних факторів. Авторами зазначено, що ряд учених та підприємців-фрілансерів обгрунтовують доцільність трансформації від фррілансу до сталої бізнес-моделі. Метою статті $\epsilon$ аналіз можливостей та наслідків використання підприємницького підходу фрілансерами для ведення власного бізнесу, визначення сили впливу використання підприємницького підходу на рівень прибутку фрілансера. Вихідну базу дослідження сформовано на основі даних наданих румунськими фрілансерами про своі фінансові результати за період 2011-2017 роки. Для перевірки висунутих гіпотез використано економетричну модель побудовану на інформаційній енергетичній моделі. Даний підхід враховує вплив зменшення ентропії у формі зростання питомої ваги доходів від підприємництва. Методологія дослідження заснована на математичному моделюванні 3 використанням структурного аналізу, що поєднує фінансові дані та статистичний аналіз для оцінювання впливу зміни обсягів доходів фрілансера, який змінив форму ведення бізнесу. Авторами наголошено, що отримані результати розширюють попередні дослідження, використовуючи теорію інформаційної енергії Оніску для специфрічного випадку підприємць-фрілансер. Перевагою адаптованого інформаційного енергетичного моделювання $є$ можливість виявлення специфіки архітектури впливу доходів фрілансерів на коригувану інформаційну енергію. Результати цього дослідження також окреслюють мотиваційні стовпи для фрілансерів, які бажають взяти участь у підприємницьких ініціативах. Перспективним напрямом подальших досліджень є розширення вибірки фрілансерів для аналізу.

Ключові слова: фріланс, підприємництво, інформаційна енергія, ентропія, економетрика.

Manuscript received: 17.07.2019

(C) The author(s) 2019. This article is published with open access at Sumy State University. 